\title{
Hereditary neutrophilia
}

INSERM

\section{Source}

INSERM. (1999). Orphanet: an online rare disease and orphan drug data base. Hereditary neutrophilia. ORPHA:279943

A rare, genetic, immune disease characterized by chronic neutrophilia, increase in the percentage of circulating CD34+ cells in peripheral blood, increase in granulocyte precursors in bone marrow and splenomegaly. Patients are predominantly asymptomatic, but may present with systemic inflammatory response syndrome with fever, dyspena, tachycardia, pleural and pericardial effusion, or myelodysplastic syndrome. 